\title{
Net Tape Graphs
}

\author{
M. El-Ghoul \\ Mathematics Department, Faculty of Science \\ Tanta University, Tanta, Egypt \\ E-mail:m.elghoul@msn.com \\ H. El-Zohny \\ Mathematics Department, Faculty of Science \\ Al-Azahar University, Cairo, Egypt \\ E-mail: elzohny_7@yahoo.com \\ M. M. Khalil \\ Mathematics Department, Faculty of Science \\ Al-Azahar University, Cairo, Egypt \\ E-mail: mona.khalil@msn.com
}

\begin{abstract}
In this paper we will introduce new type of graphs, when vertices of these graphs are appearance like line and edges of these graphs are appearance like tape or ribbon and when this tape has infinity holes we call these graph (net tape graph or net graph). We introduce types of representation of the new graph by the adjacent and the incidence of matrices, and we will discuss their transformations.
\end{abstract}

AMS Subject Classification 2000: 51H10, 57N10.

Keywords: Graphs, Transformations

\section{Definitions and background}

Abstract graphs: An abstract graphs $G$ is a diagram consisting of a finite non empty set of the elements, called "vertices" denoted by $V(G)$ together with a set of unordered pairs of these elements, called "edges" denoted by $E(G)$. The set of vertices of the graph $G$ is called "the vertex - set of $G$ " and the list of edges is called "the edge - list of $G$ " [Gibbons, 1995; Giblin, 1977].

Adjacency and incidence: let $v$ and $w$ be vertices of a graph. If $v$ and $w$ are joined by an edge $e$. then $v$ and $w$ are said to be adjacent. Moreover, $v$ and $w$ are said to be incident with $e$, and $e$ is said to be incident with $v$ and $w$ [Wilson, 1972].

The adjacency matrix: let $G$ be a graph without loops, with $n$-vertices labeled $1,2,3, \ldots, n$. The adjacency matrix $A(G)$ is the $n \times n$ matrix in which the entry in row $i$ and column $j$ is the number of edges joining the vertices $i$ and $j$ [Wilson, 1972].

The incidence matrix: let $G$ be a graph without loops, with $n$-vertices labeled $1,2,3, \ldots n$ and $m$ edges labeled $1,2,3, \ldots$, $m$. the incidence matrix $I(G)$ is the $n \times n$ matrix in which the entry in row $i$ and column $j$ is $l$ if vertex $i$ is incident with edge $j$ and 0 otherwise [Gross, 1987; Wilson, 1990].

Isometric folding: let $M$ and $N$ be smooth connected Riemannian manifolds of dimensions $m$ and $n$ respectively such that $m \leq n$. A map $f: M \longrightarrow N$ is said to be an isometric folding of $M$ into $N$ iff for every piecewise geodesic path $\gamma: J \longrightarrow M$ the induced path $f$ o $\gamma: J \longrightarrow N$ is a piecewise geodesic and of the same length as $\gamma$ [Robertson, 1977].

Folding and unfolding of graph:

(a) Let $f: G \rightarrow \bar{G}$ be a map between any two graphs $G$ and (not necessary to be simple) such that if $(u, v) \in G,(f(u), f(v))$ $\in \bar{G}$. Then $f$ is called a "topological" of $G$ to provided that $d(f(u), f(v)<d(u, v)$ [Giblin, 1977].

(b) Let $g: G \rightarrow \bar{G}$ be a map between any two graphs $G$ and (not necessary to be simple)such that if (u,v) $\in G$, $(g,(u), g(v)) \in \bar{G}$. Then $g$ is called a "topological unfolding" of to $\bar{G}$ provided that $d(g(u), g(v))>d(u, v)[$ El-Ghoul].

Retracts: A subset $A$ of a topological space $X$ is called a "retract" of $A$ if there exists a continuous map $r: X \rightarrow A$ (called a retraction) such that $r(a)=a \forall a \in A$, where $A$ is closed and $X$ is open. In other words, a retraction is a continuous map of a space onto a subspace leaving each point of the subspace fixed [El-Ghoul, 2004]. 


\section{Main Results}

Now we will define and discuss the tape net graph and some transformations on this new graph, and that will be represented by matrices.

\subsection{Definition}

The net tape graph $G$ is a diagram consisting of a finite non empty set of the elements with "line or curve" shape called "vertices" denoted by $V(G)$ together with elements, with "tape" shape called "edges" denoted by $E(G)$, and their edges contain an infinity holes with different shapes .

The matrix representation of geometric tape graph considers the geometric graph $G\left(v^{0} v^{1}\right)$ see Fig. (1).

It's adjacent and incidence matrices are:

$A(G)=\left[\begin{array}{ll}0 & 1 \\ 1 & 0\end{array}\right]_{2}, I(G)=\left[\begin{array}{l}1 \\ 1\end{array}\right]_{2}$.

The matrix representation of geometric a hole tape graph considers the geometric graph $G\left(v^{0} v^{1}\right)$ see Fig. (2).

It's adjacent and incidence matrices are:

$A(G)=\left[\begin{array}{ll}0^{0} & 1^{1} \\ 1^{1} & 0^{0}\end{array}\right]_{2}, I(G)=\left[\begin{array}{l}1^{1} \\ 1^{1}\end{array}\right]_{2}$.

Where a superscript 0,1 mean the number of holes.

The matrix representation of geometric two holes in tape graph considers the geometric graph $G\left(v^{0} v^{1}\right)$ see Fig. (3).

It's adjacent and incidence matrices are:

$A(G)=\left[\begin{array}{cc}0^{0} & 1^{2} \\ 1^{2} & 0^{0}\end{array}\right]_{2}, I(G)=\left[\begin{array}{c}1^{2} \\ 1^{2}\end{array}\right]_{2}$.

Where a superscript 0,2 mean the number of holes.

The matrix representation of geometric three holes in tape graph considers the geometric graph $G\left(v^{0} v^{1}\right)$ see Fig. (4).

It's adjacent and incidence matrices are:

$A(G)=\left[\begin{array}{ll}0^{0} & 1^{3} \\ 1^{3} & 0^{0}\end{array}\right]_{2}, I(G)=\left[\begin{array}{c}1^{3} \\ 1^{3}\end{array}\right]_{2}$.

Where a superscript 0,3 mean the number of holes.

The matrix representation of geometric $n$ holes in tape graph considers the geometric graph $G\left(v^{0} v^{1}\right)$ see Fig. (5).

Its adjacent and incidence matrices are:

$A(G)=\left[\begin{array}{ll}0^{0} & 1^{n} \\ 1^{n} & 0^{0}\end{array}\right]_{2}, I(G)=\left[\begin{array}{l}1^{n} \\ 1^{n}\end{array}\right]_{2}$.

Where a superscript $0, n$ mean the number of holes.

We can see that Fig. (5) is new type of graph called a finite net tape graph.

\section{Theorem 1:}

If the number of holes is then we obtain another new type it is 1-dimensions graph it depend on a method which I divided these holes see Fig. (6), Fig. (7) and Fig. (8).

As we show above all these figures represent new type of one dimension graph called "filter graph". Or any graph homeomorphic to Fig. (8).

\section{Lemma 1:}

The limit of stretching net tape graph is a filter graph.

The matrix representation of geometric $\infty$ holes in the tape graph considers the geometric graph $G\left(v^{0} v^{1}\right)$.

Its adjacent and incidence matrices are:

$A(G)=\left[\begin{array}{cc}0^{0} & 1^{\infty} \\ 1^{\infty} & 0^{0}\end{array}\right]_{2}, I(G)=\left[\begin{array}{l}1^{\infty} \\ 1^{\infty}\end{array}\right]_{2}$.

$A(G)={ }_{n} \lim _{\infty}\left[\begin{array}{ll}0^{0} & 1^{n} \\ 1^{n} & 0^{0}\end{array}\right]_{2}=\left[\begin{array}{cc}0^{0} & 1^{\infty} \\ 1^{\infty} & 0^{0}\end{array}\right]_{2}, I(G)={ }_{n} \lim _{\infty}\left[\begin{array}{l}1^{n} \\ 1^{n}\end{array}\right]_{2}=\left[\begin{array}{l}1^{\infty} \\ 1^{\infty}\end{array}\right]_{2}$. 
Where a superscript $0, \infty$ mean an infinite holes. We will introduce types of representation of this graphs by the adjacent and the incidence of matrices, and when their connected.

\section{a-Connected finite graph}

It's adjacent and incidence matrices are:

$A(G)=\left[\begin{array}{ccc}0^{0} & 1^{n} & 0^{0} \\ 1^{n} & 0^{0} & 1^{n} \\ 0^{0} & 1^{n} & 0^{0}\end{array}\right]_{2}, I(G)=\left[\begin{array}{cc}1^{n} & 0^{0} \\ 1^{n} & 1^{n} \\ 0^{0} & 1^{n}\end{array}\right]$.

See Fig.(9).

\section{b- Connected infinite net graph}

It's adjacent and incidence matrices are:

$A(G)=\left[\begin{array}{ccc}0^{0} & 1^{\infty} & 0^{0} \\ 1^{\infty} & 0^{0} & 1^{\infty} \\ 0^{0} & 1^{\infty} & 0^{0}\end{array}\right]_{2}, I(G)=\left[\begin{array}{cc}1^{\infty} & 0^{0} \\ 1^{\infty} & 1^{\infty} \\ 0^{0} & 1^{\infty}\end{array}\right]$.

\subsection{Folding of geometric net tape graph}

When we folding the net tape graph into itself, there are three types to folding it such that:

During the processing of folding the holes become above each other completely (holes are regular), and in this case we decreasing the area of graph and the numbers of holes see Fig. (10).

During the processing of folding the holes become above each other but not completely (holes are irregular), and in this case we decreasing the area of graph and holes but also the area of holes are increasing see Fig. (11).

During the processing of folding the holes absolutely don't become above each other, and in this case we obtain an ordinary tape graph with no holes see Fig. (12).

\section{Theorem 2:}

The limit of foldings of infinite net tape graph $G$ into itself is a finite net tape graph or a simple graph.

\section{Proof:}

case(1): let $f_{1}: G \longrightarrow G$, such that $f$ net graph=net graph then $f_{2}: f_{1}(G) \longrightarrow f_{1}(G)$ is a net tape graph,..., $f_{n}: f_{n-1}\left(f_{n-i}(G)\right) \longrightarrow f_{n-1}\left(f_{n-i}(G)\right)$ is a net tape graph, ${ }_{n} \lim _{\infty} f_{n}(G)=K, K=$ one net tape see Fig.(13).

Case (2): Let $\overline{f_{1}}: G \longrightarrow(G)$, such that $\overline{f_{1}}$ (any net tape)= net tape graph of less than area.Then $\overline{f_{2}}(T)=T^{1}, \overline{f_{3}}\left(T^{1}\right)=T$ $2, \ldots, \overline{f_{n}}\left(T^{n-2}\right)=T^{n-1}, \ldots,{ }_{n} \lim _{\infty} \overline{f_{n}}\left(T^{n-2}\right)=$ edge of 1-dimension see Fig.(14).

\section{Theorem 3:}

The folding of 1- dimension filter graph is a filter graph not a simple graph.

\section{Proof:}

Folding filter graph horizontally and the holes in the filter graph are divided horizontally. Let $f f_{1}: f(G) \longrightarrow f(G)$, then $f$ ${ }_{1}\left(L_{i}\right)=\left(L_{j}\right)$ where $L_{i}, L_{j}$, are two fibers with the same length $f_{2}\left(L_{j}\right)=\left(L_{k}\right), \ldots . . f_{n}$, see Fig. (15).

Or Folding filter graph vertically and the holes in the filter graph are divided vertically. Let $g_{1}: f(G) \longrightarrow f(G)$, then $g_{1}\left(L_{i}\right)=\left(L_{j}\right)$ where $L_{i}, L_{j}$, are two fibers with the same altitude $g_{2}\left(L_{j}\right)=\left(L_{k}\right), \ldots . . g_{n}$, see Fig. (16).

\subsection{Retraction of geometric net tape graph}

The retraction a net tape graph: 1-from the middle we obtained a connected null tape graph the vertices and edges have the same dimension, it's adjacent and the incidence matrices are given by:

$A(G)=\left[\begin{array}{l}1 \\ 1\end{array}\right], I(G)=\left[\begin{array}{l}1 \\ 1\end{array}\right]$,see Fig.(17).

2- From the rim of edges we obtained a simple graph, it's adjacent and the incidence matrices will be:

$A(G)=\left[\begin{array}{ll}0 & 1 \\ 1 & 0\end{array}\right], I(G)=\left[\begin{array}{l}1 \\ 1\end{array}\right]$, see Fig.(18).

\section{Applications:}

1- A fishnet is a net tape graph. 
2- A sponge is a net tape graph.

3-TV.screen and the printer is afilter graph.

4- Sbider web is a filter graph.

\section{References}

El-Ghoul, M, El-Zohny, H, Khalil, M, M. (2010). On tape graph. Journal of mathematics research, Vol. 2, No. 4. Issn: 1916-9795, Canada, Nov.2010. Under press.

El-Ghoul, M. Unfolding of graph and uncertain graph. The Australian mathematics journal, sandy Bay 7006, Tasmania, Australia.

El-Ghoul, M, El-Ahmady, A.and Rafat, H. (2004). Folding-retraction of chaotic dynamical manifold and the VAK of vacuum fluctuation. chaos, Solutions and Fractals, UK, 20:209-217.

Gibbons, A. (1995). Algorithmic graph theory. Cambridge University Press, Cambridge, UK.

Giblin, P.J. (1977). Graphs, surfaces and homology, an introduction to algebraic topology. Chapman and Hall. Ltd, London 1977.

Gross, J.L. and Tucker, T.W. (1987). Topological graph theory. Jon Wiley \& Sons, Inc, Canada 1987.

Robertson, S.A. (1977). Isometric folding of Riemannian manifolds. Proc.Fac.Roy.Soc.Edinburgh, 77, $275-284$.

Wilson, R.J. (1972). Introduction to graph theory. Olivar\& Boyed, Edinburgh.

Wilson, R.J. and Watkins, J.J. (1990). Graphs, an introductory approach, a first course in discrete mathematics. Jon Wiley \& Sons Inc, Canada.

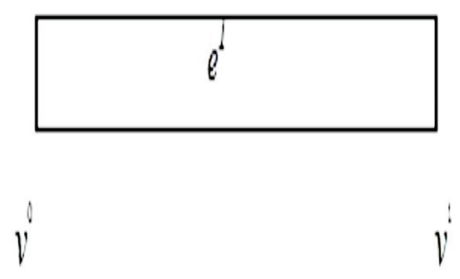

Figure 1.

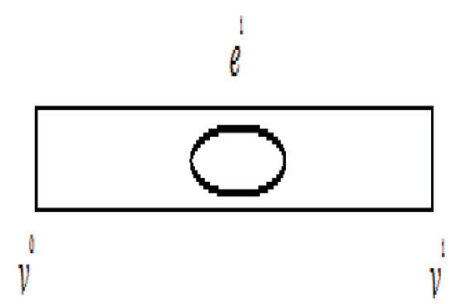

Figure 2.

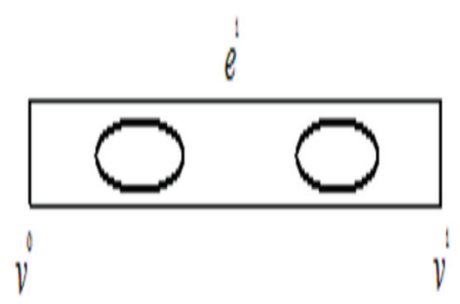

Figure 3.

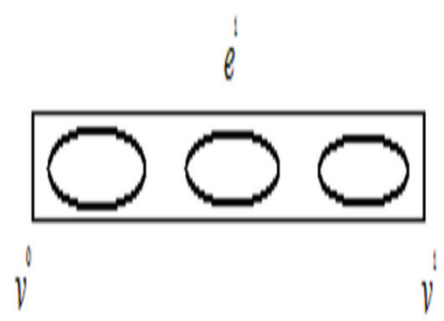

Figure 4. 


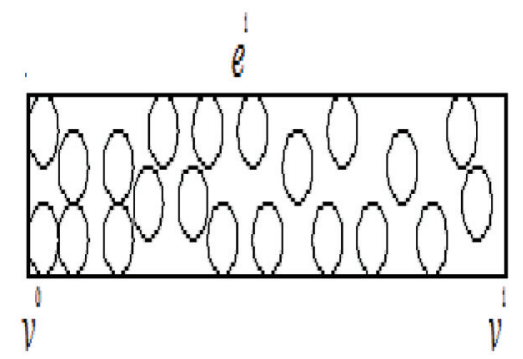

Figure 5.

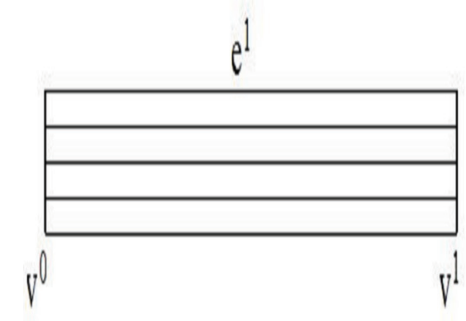

Figure 6.

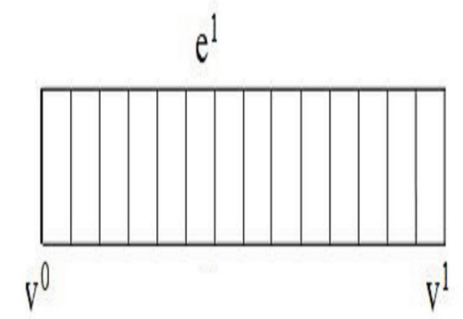

Figure 7.

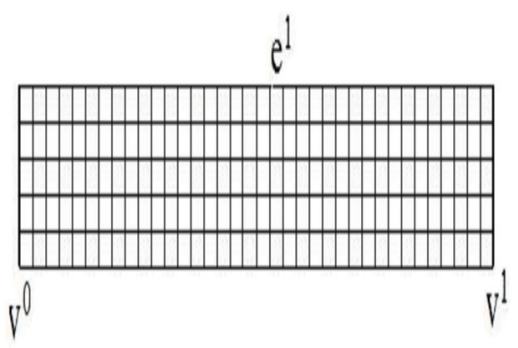

Figure 8.

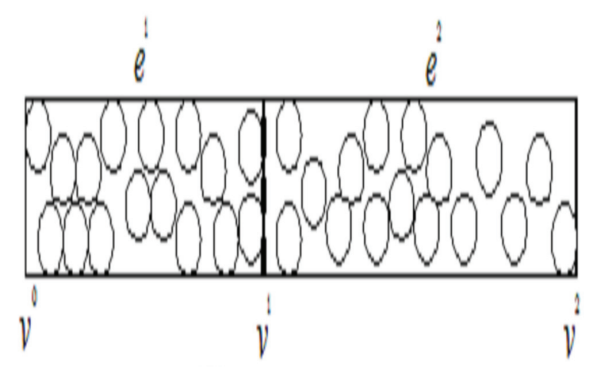

Figure 9. 


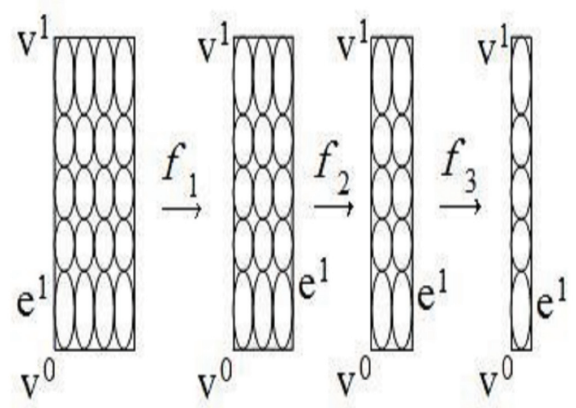

Figure 10.

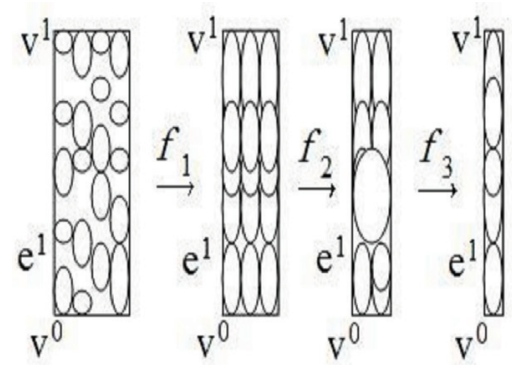

Figure 11.

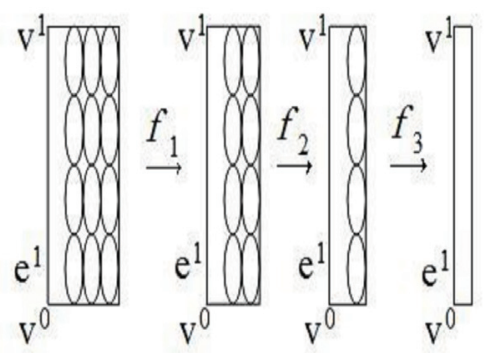

Figure 12.

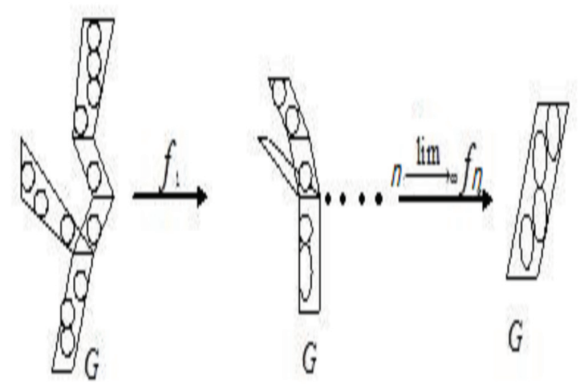

Figure 13.

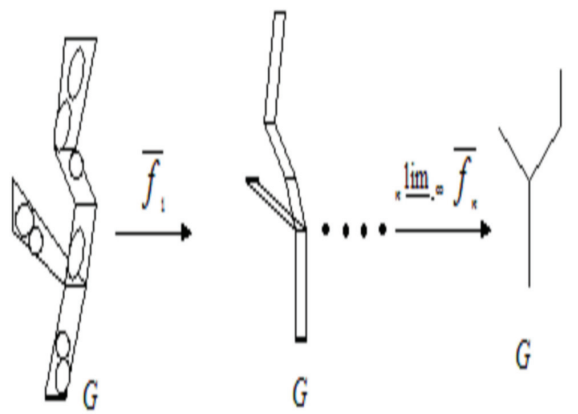

Figure 14. 

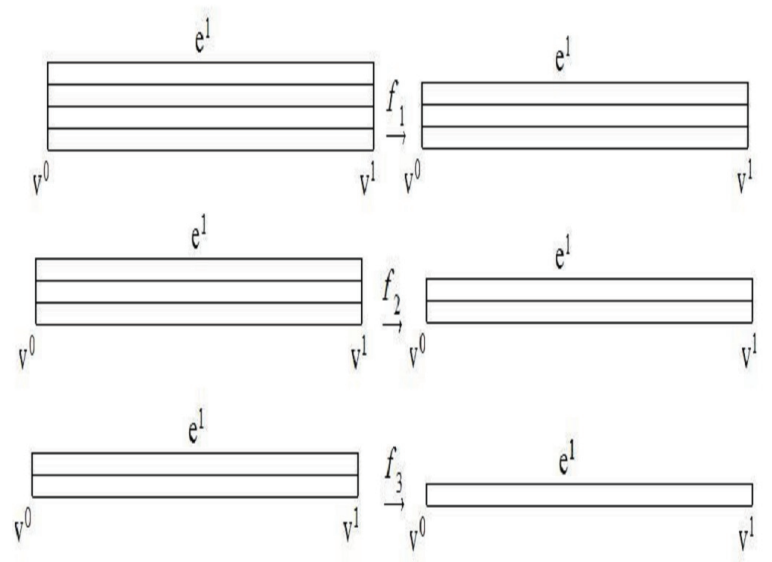

Figure 15.

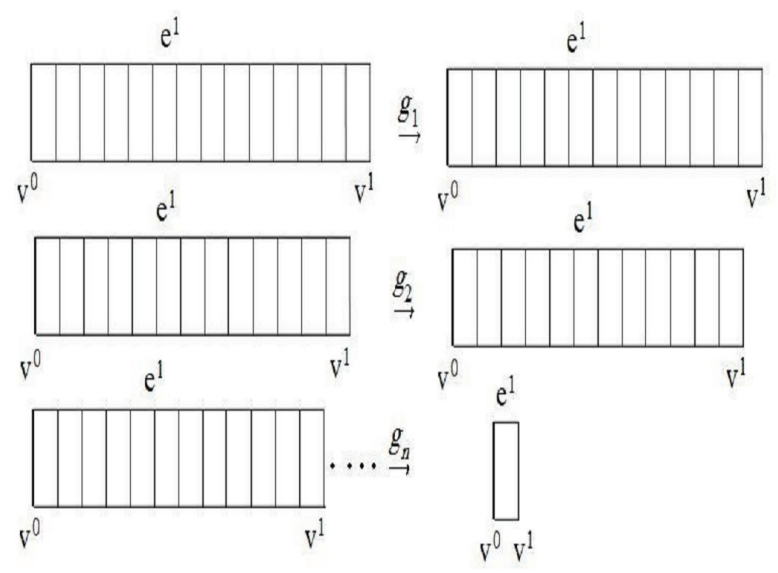

Figure 16.

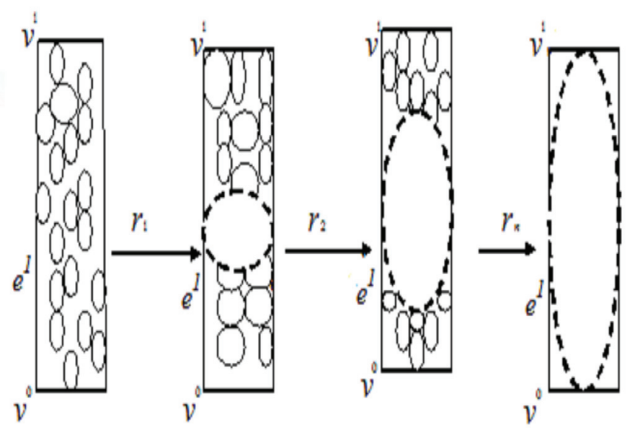

Figure 17.

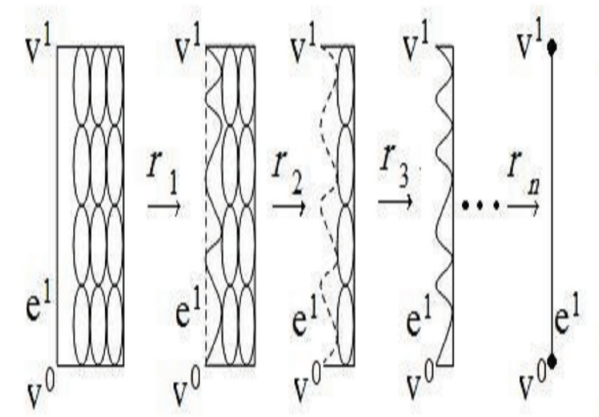

Figure 18. 